\title{
Tackling Childhood Obesity in Hong Kong
}

\author{
Angie Ho Yan Lam* \\ Lecturer, The University of Hong Kong, Hong Kong \\ *Corresponding author: Angie Ho Yan Lam, Lecturer, The University of Hong Kong, Hong Kong

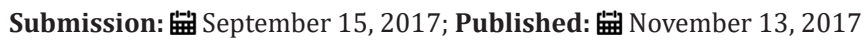

\begin{abstract}
Childhood overweight and obesity is one of the major problems in Hong Kong. Overweight and obese children have a high risk of developing chronic diseases and psychological impacts. The determinants to children obesity in the Chinese context include Chinese traditional culture, parenting knowledge and practice, sedentary lifestyle and high academic attainment. Conceptual framework is formulated to guide the idea of Family-School Partnership intervention in Hong Kong Context.
\end{abstract}

\section{Editorial}

Childhood overweight and obesity is not only a local problem in Hong Kong but also a global concern WHO [1], Centre for Health Protection [2]. The age standardized prevalence of overweight and obesity in school students aged 2 to 19 years increased from $8.3 \%$ to 13.2\% between 1980 and 2013 globally Fleming et al. [3]. In Hong Kong, the prevalence of overweight and obesity among primary school students increased from $16.1 \%$ in 1995 to $20 \%$ in 2013 Centre for Health Protection [2]. Overweight and obese children have a high risk of developing chronic diseases in adulthood. Compared with children classified as not overweight, obese children were more likely to have metabolic diseases in adulthood, such as diabetes mellitus (Relative Risk (RR): 5.4), hypertension (RR: 2.7), increased in triglyceride (RR: 3.0), and atherosclerosis (RR: 1.7) Juonala et al. [4].

Research also demonstrated that obese children were more likely to have psychological problems such as activity restrictions, internalizing problems (worthless/inferior, sad/depressed, low self-esteem, and social withdrawal), externalizing problems (argumentative, cruel, disobedient, stubborn and irritable), and grade repetition Fleming et al. [3]. Chinese traditional child care beliefs contribute to child obesity in Hong Kong context. Parents and careers believed in "good appetite is kinds of blessing". There is a widespread traditional belief that excess body fat represents health, prosperity and good parenting $\mathrm{Wu}$ [5]. Besides, parental knowledge and practice are crucial factors contributing to childhood obesity in Hong Kong. Parents and careers used to provide tasty food as a way of love and appraisals. Some parents showed misconception about healthy diet and child obesity. They believed that the problem of obesity would be resolved when the children grew up Li et al. [6]. Furthermore, it is highly academically orientated in Hong Kong, and admission to prestigious secondary school is extremely competitive.
Students are exhausted by the intense amounts of work and study, both in class and after class, resulting in sedentary lifestyle with ignoring the importance of physical activity and school life balance Li et al. [7]. Childhood obesity prevention programs should be implemented at an early stage of child development. Even though Department of Health organizes various programs, such as EatSmart@school.hk Campaign, to cultivate a healthy lifestyle among school children and preschool children. Those programs mainly implement school-based activities without emphasizing the collaboration between families and schools.

\section{Conceptual framework}

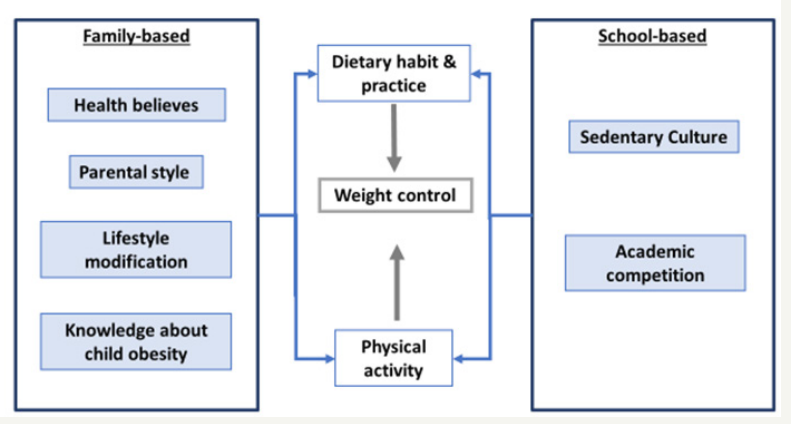

Figure 1: Concept framework for childhood obesity prevention program in Hong Kong context.

Conceptual framework is formulated to serve as a guide for the family-school partnership intervention (Figure 1). Family school Partnership is an evidence-based approach to reinforce active collaboration between schools and families, and share the responsibility for preventing children obesity. The obesity stakeholders including schools, homes, communities and clinical settings should be involved in the program. As Chinese traditional 
cultural believes highly contributes to child obesity, the intervention should be culturally sensitive to meet the different expectation of Asian families. Through family-school partnership interventions focused on collaboration among different stakeholders, the entire community concrete a favorable environment and culture which are supportive of healthy eating and physical activity to prevent subsequent childhood obesity.

\section{References}

1. WHO (2016) Childhood overweight and obesity, World Health Organization, Geneva, Switzerland.

2. (2015) Healthy Non Communicable Disease Watch-Weight Healthy Kids, Centre for Health Protection and Department of Health.
3. Ng M, Fleming T, Robinson M, Thomson B, Graetz N, et al. (2014) Global, regional, and national prevalence of overweight and obesity in children and adults during 1980-2013: a systematic analysis for the Global Burden of Disease Study 2013. Lancet 384(9945): 766-781.

4. Juonala M, Magnussen CG, Berenson GS, Venn A, Burns TL, et al. (2011) Childhood adiposity, adult adiposity, and cardiovascular risk factors. N Engl J Med 365(20): 1876-1885.

5. Wu Y (2006) Overweight and obesity in China. BMJ 333(7564): 362-363.

6. Li B, Adab P, Cheng KK (2015) The role of grandparents in childhood obesity in China - evidence from a mixed methods study. Int J Behav Nutr Phys Act 12: 91.

7. Li B, Lin R, Liu W, Chen J, Liu W, et al. (2017) Differences in perceived causes of childhood obesity between migrant and local communities in China: A qualitative study. PLoS ONE 12(5): e0177505. 